\title{
Numerical Studies on High-Velocity Impact Welding: Smoothed Particle Hydrodynamics (SPH) and Arbitrary Lagrangian-Eulerian(ALE)
}

\author{
Ali Nassiri ${ }^{1,2, *}$ and Brad Kinsey ${ }^{3}$
}

\begin{abstract}
The manufacturing industry often relies on numerical simulations to reduce the number of trial-anderror iterationsrequired during the process development to reduce costs. However, this can bedifficultin high strain rate manufacturing processes. For instance, in high-velocity impact welding, extremely high plastic strain regions develop. Thus, a traditional pure Lagrangian analysis is not able to accurately model the process due to excessive element distortion near the contact zone.In this paper, numerical simulations were conducted using Smoothed Particle Hydrodynamics (SPH) and Arbitrary Lagrangian-Eulerian (ALE) methods to investigatea high-velocity oblique impact between two metal plates. While the preliminary results show that the two methods were capable of modeling the process,a trade-off between the computational costand full prediction of the critical process parameters should beconsidered for industrial applications.
\end{abstract}

Keywords:High-Velocity Impact Welding, Smoothed Particle Hydrodynamics, Arbitrary Lagrangian-Eulerian

\section{Introduction}

In order to meet the increasingly ambitious Corporate Average Fuel Economy (CAFE) standards and reduce $\mathrm{CO}_{2}$ emissions, there is a growing interest in creating lightweight vehicles. One approach relies on the use of lower-density materials such as aluminum, magnesium, and advanced composites[1,2]. These materials can have increased strength/density ratios and improved section

1. Department of Materials Science and Engineering, The Ohio State University,2041 College Road N, Columbus, OH 43210, USA.

2. Center for Design and Manufacturing Excellence, The Ohio State University, 1314 Kinnear Road, Columbus, OH 43212, USA.

3. Department of Mechanical Engineering, University of New Hampshire, 33 Academic Way, Durham, NH 03824, USA

Corresponding author. Tel: +1-614-688-2594, Email: nassiri.3@osu.edu 
moduli. The philosophy behind this approach is to use each material where it provides the greatest value. For instance, magnesium alloys with the lowest density can be used where energy abortion is not required. Creation of such structures requires joining dissimilar metals. Due to differences in melting points and the tendency to form brittle intermetallic compound (IMCs), many dissimilar metals pairs cannot be joined with traditional fusion-based welding techniques. Solid state welding techniques such as High-Velocity Impact Welding (HVIW), Friction Stir Welding, and Ultrasonic Welding are feasible methods for joining dissimilar materials. Due to the short cycle time of the process, the ability to perform the process over varying length scales, and the wide variety of dissimilar metal combinations able to be joined (more than 260 combinations [3]), HVIW has received significant attentionover the past fewdecades [4-6].

HVIW methods include techniques such as EXplosive Welding (EXW), Magnetic Pulsed Welding (MPW) and Vaporizing Foil Actuator Welding (VFAW). In all of these techniques, the flier workpiece first accelerates to a high velocity with a significant kinetic energy before it strikes another workpiece which is stationary. The difference between the aforementioned methods lies in the way the flier workpiece is accelerated toward the stationary one [3,5,7].A fundamental understanding of HVIW will allow the design space to be further investigated without time-intensive and costly experimental trials while also providing details to fundamentally understand the embedded process parameters. For instance, a wavy interface morphology is often observed during HVIW processes, provided that the impactvelocity and angle are sufficient and correct, respectively(see Fig. 1).If such a morphology occurs below the melting points of the materials, a successful weld is assumed [7-9].

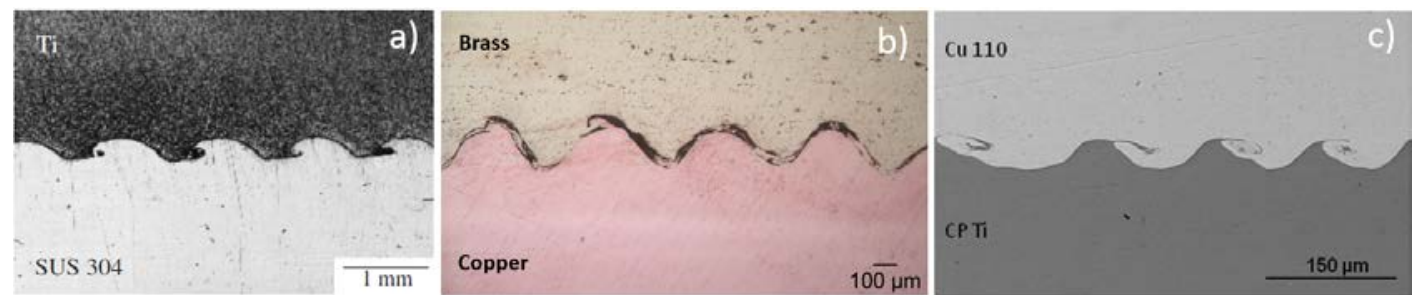

Figure 1: Wavy morphology during HVIW in a) EXW [10], b) MPW [11], c) VFAW [12]

Due to the highly coupled and dynamic nature of the HVIW processes, numerical simulations are very challenging, especially near the welding zone where large deformations occur. Thus, a traditional Lagrangian method where the mesh is fixed to the workpiece geometry fails as a results of excessive element distortion (see Fig. 2).Recent advancements in finite element analysis (i.e., different algorithms and improved contact mechanics) have produced models that may capture the essentials of structure development in this process.

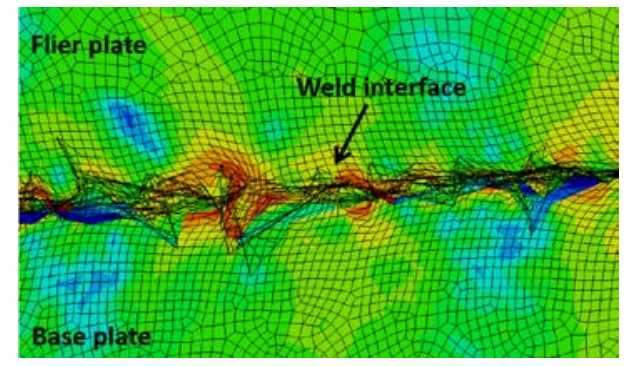

Figure 2: Distorted mesh at the weld interface in pure Lagrangian simulation [7] 
Commonly, pure Eulerian analysis is used to address such concerns [13]. In this method, nodes are fixed and the material flows through the elements. This method has some disadvantage though when free surfaces with large displacements have to be taken in account. Also, the storage and computational cost of anEulerian analysis is higher than the traditional pure Lagranigan analysis.

An Arbitrary Lagrangian-Eulerian approach (ALE) is another alternative [7]. The ALE method is a technique that combines the features of Lagrangian and Eulerian analyses within the same mesh to maintain a high mesh quality during simulations involving large deformations. ALE consists of two fundamental tasks at each time increment: 1) creating a new mesh and 2) remapping the solution variables from the old mesh to the new mesh. A new mesh is created at a specified frequency for each adaptive mesh domain. The mesh is determined by iteratively sweeping over the adaptive mesh domain and moving nodes to "smooth" the mesh. The computational cost of adaptive meshing depends on the frequency of remeshing, the number of elements, and the size of the adaptive mesh domain. The ALE method has been used in many research efforts including the study of a solid-fluid interaction [14], large deformation analysis [15], and metal cutting-forming process [16].

During the last decade, the Smoothed Particle Hydrodynamics (SPH) methodhas received significant attention. SPH is a meshless method where collection of points are used to represent a continuum body. The ability to handle severe distortions allows the SPH technique to be applied to problems that historically have been reserved for pure Eulerian approaches. Recently, with the advancement in mathematical and numerical modeling and progress in hardware capability and parallel processing, the SPH method has been used in many fields of research including ballistic impact [17], multi-fluid problems that may involve the motion of rigid and elastic bodies [18], nonNewtonian fluids [19], virtual surgery [20],and chemical precipitation from fluids moving through fractured media[21].

In this paper, in order to further understand the science behind HVIW processes and to compare numerical methods, two numerical simulations methods were used. First, a SPH model of two impacting plates with an initial offset angle of 7 degrees was created. Using SPH method, metal jet emission were investigated which previously was impossible. To understand the relative benefits and drawback of various numerical simulation methodologies, the results then were compared with the ALE method. This will provideconfidence in the models created. Finally, the numerical results were compared with an experimental test.

\section{Numerical Simulation Model}

The 2D numerical simulations were carried out in the non-linear Abaqus/Explicit 6.13 software package to simulate an impact between two Al6061-T6 plates. The dimensions of the flier and base plates are $12 \mathrm{~mm}$ by $2 \mathrm{~mm}$ and $15 \mathrm{~mm}$ by $3 \mathrm{~mm}$ respectively (see Fig. 3 ). The lengths assured a steady state phenomenon was achieved, and the thicknesses assured interface effects were captured.The FE analyses were conducted by imposing a flier plate velocity normal to the interface (i.e., impact velocity, $\left.V_{F}=350 \mathrm{~m} / \mathrm{s}\right)$ and fixing the initial angle between the flier and base plates $\left(\alpha=7^{\circ}\right)$ as initial conditions. The left side and bottom of the base plate were fixed as boundary conditions. The impact velocity produces a collision (or shear) velocity $\left(\mathrm{V}_{\mathrm{C}}\right)$. In thesimulations, the contact between the two

plates was defined as surface-to-surface with a penalty method and finite sliding formulation.Friction was defined with a penalty method, and a coulomb friction coefficient of $\mu=0.15$ was used. 


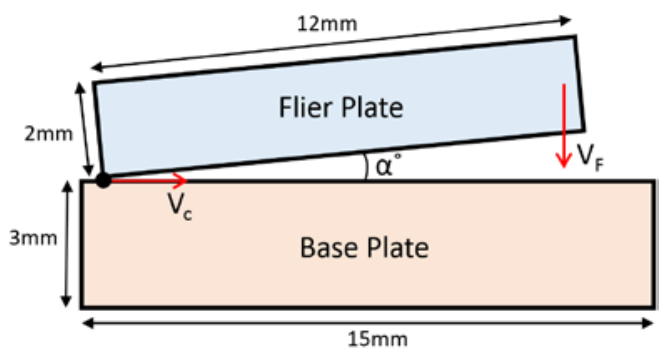

Figure 3: Schematic of Finite Element Model

The material behavior was modelled using a Johnson-Cook constitutive relation:

$$
\sigma=\left(A+B \varepsilon_{e f f}{ }^{n}\right)(1+C \ln \dot{\varepsilon})\left(1-T^{* m}\right)
$$

where $\sigma$ is the flow stress, $\varepsilon_{e f f}$ is the effective plastic strain, $\dot{\varepsilon}=\frac{\dot{\varepsilon}_{e f f}}{\dot{\varepsilon}_{0}}$ is the plastic strain rate, $T^{*}=$ $\frac{T-T_{\text {room }}}{T_{\text {melt }}-T_{\text {room }}}$ is the homologous temperature, and $A, B, C, n$ and $m$ are material parameters. At the melting temperature(i.e., $T^{*}=1$ ) the stress approaches zero for all strain and strain values. The Johnson-Cook parameters for Al6061-T6 are given in Table 1[22].Due to the high strain rate, adiabatic heating was assumed with an inelastic heat fraction of $0.9[23]$. This term characterizes the fraction of plastic work converted into heat which is usually assumed to be a constant although it can be strongly depend on strain and strain rate.

\begin{tabular}{|c|c|c|c|c|c|c|c|}
\hline $\begin{array}{c}\rho \\
\left(\mathrm{kg} / \mathrm{m}^{3}\right)\end{array}$ & & & \multicolumn{2}{|c|}{$v$} & $\begin{array}{c}k \\
(W / m k)\end{array}$ & \multicolumn{2}{|c|}{$\begin{array}{c}\mathrm{C} \\
\left(\mathrm{J} / \mathrm{kg}^{\circ} \mathrm{C}\right)\end{array}$} \\
\hline 2700 & \multicolumn{2}{|c|}{70} & \multicolumn{2}{|c|}{0.279} & 154 & \multicolumn{2}{|c|}{860} \\
\hline $\begin{array}{c}\text { A } \\
\text { (MPa) }\end{array}$ & $\begin{array}{c}\text { B } \\
\text { (MPa) }\end{array}$ & C & n & m & $\dot{\varepsilon}_{0}$ & $\begin{array}{l}\mathbf{T}_{\text {melt }} \\
\left({ }^{\circ} \mathbf{C}\right)\end{array}$ & $\begin{array}{l}\mathbf{T}_{\text {room }} \\
\left({ }^{\circ} \mathbf{C}\right)\end{array}$ \\
\hline 289 & 108 & 0.011 & 0.42 & 1.34 & 1 & 653 & 23 \\
\hline
\end{tabular}

Table 1: Material properties and Johnson-Cook parameters for Al6061-T6

\subsection{Smoothed Particle Hydrodynamics (SPH)}

SPH is a fully Lagrangian particle-based method where a collection of particles, instead of classical elements in a traditional finite element simulation, are used to represent a given body.These particles have a spatial location in the numerical model. A physical quantity of any particle is obtained by summing the relative properties of a set of neighboring particles, denoted by subscript $j$, for which the Kernel function (see Fig. 4), $W$, is not zero [24].

$$
f(r) \cong \sum_{j} \frac{m_{j}}{\rho_{j}} f_{j} W\left(\left|r-r_{j}\right|, h\right)
$$

In Eq. (2), $f(r)$ shows the field variable, $r$ is the location of the particles, $m_{j}$ and $\rho_{j}$ are the associate mass and density of the particles respectively, and $h$ indicates the smoothing length and 
determines how many particles influence the interpolation for a particular point. In this method, close particles contribute more than distant particles. The Kernel is analogous to the shape function in the finite element method, but does not require connectivity between particles. Hence, these particles are able to move relative to each other in the domain of the simulation, thus allowing the jetting phenomenon observed during HVIW to be numerically predicted.

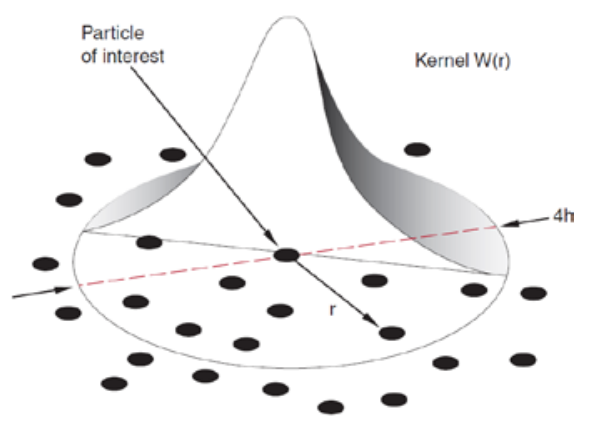

Figure 4: The principle of SPH Kernel function [24]

The particle size plays a significant role in the simulation due to the computational time required, as well as visualizing the jetting particles and interface profiles. In this study, the particle size was set to be $5 \mu$ mand a total of 440,000 particles were used in the model.

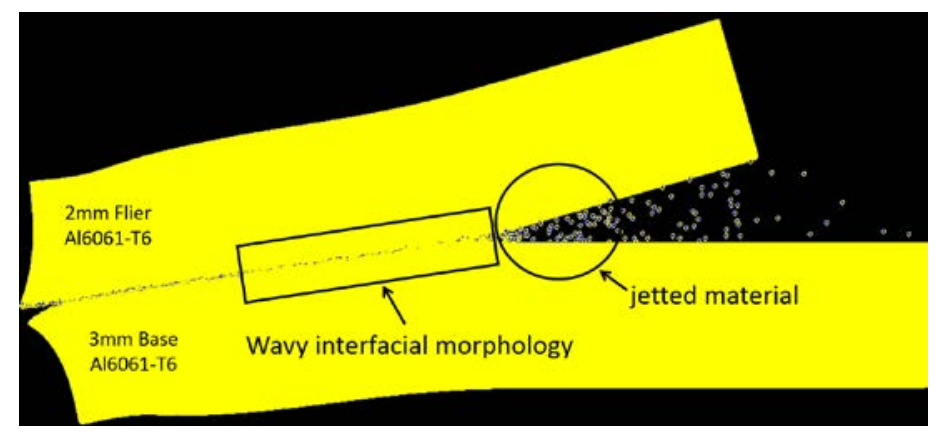

Figure 5:SPH prediction of two plates after $30 \mu \mathrm{s}$

Fig. 5 shows the two platesapproximately30 $\mu$ safter initial impact for the case where the impact velocity, $V_{F}$, and impact angle, $\alpha$, were $350 \mathrm{~m} / \mathrm{s}$ and $7^{\circ}$ respectively. Fig. 6 providesa close-up of the interface. As is clear,the jetting effect is evident. Researchers usually consider jetting as a prerequisite for welding to occur[25]. Note that the jetted materials could include the entire oxide layer which is typically 2-10nm thick for Aluminum alloys [26]. Removal of the oxide layer would produce a more fracture tough weld.Also as evident in Fig. 6, SPH modeling is able to predict the wavy morphology at the interface as well. Additionally, since both the flier and base plates have the same material properties (e.g., density), the composition of the jetis almost the same. However, it is conceivable that the composition of the jet will be governed by the degree of relative density difference between the two plates. When the density difference is large, the jet will mainly be composed of the plate with the lower density. However, whether the model is accurately capturing the jetting and material behavior is unknown. 


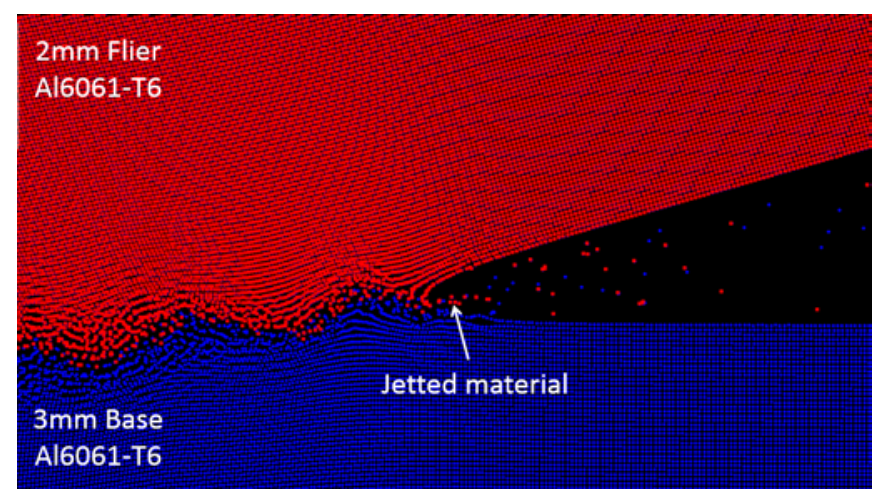

Figure 6: Jetting phenomenon during impact welding of two Al6061-T6 plates

Fig. 7 shows the collision velocity field near the contact zone. Clearly, a symmetric localized high velocity zone was produced at the contact point in which the material close to the collision point of both the flier and base plates moves with the same velocity.The high velocity field is responsible for the plastic deformation and leads to a shear instability discussed inReferences [8,9]. This in turn produced a high value of plastic strain and shear stress at the interface.Also, Fig. 7 shows the velocity of the collision point and jetted material are approximately $1200 \mathrm{~m} / \mathrm{s}$ and $>2000 \mathrm{~m} / \mathrm{s}$ respectively. The captured collision velocity, i.e., $1200 \mathrm{~m} / \mathrm{s}$, is higher than the minimum velocity required to createa wavy morphology at the interface according to the weldability window discussed in [7].

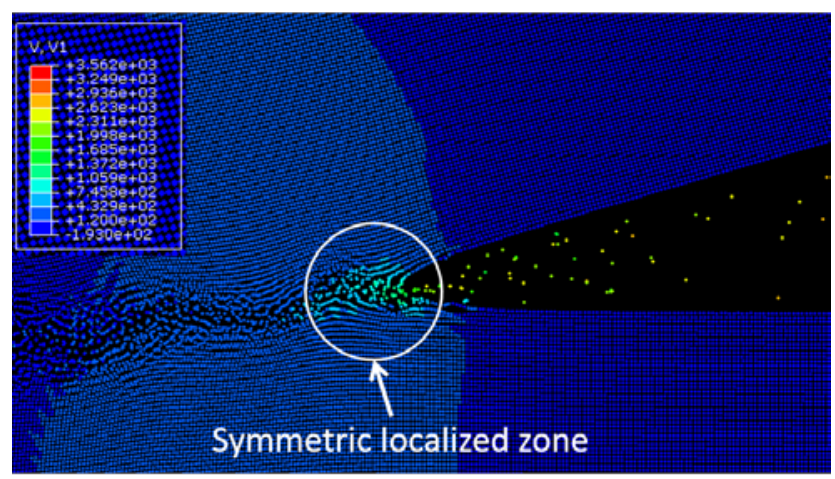

Figure 7:Predicted symmetric collision velocity distribution

Fig. 8 shows the shear stress where different signs were produced at the interface. At the collision point, the shear stress, $370 \mathrm{MPa}$, exceeded the shear yield stress of the Al6061-T6 (see Table 1). 


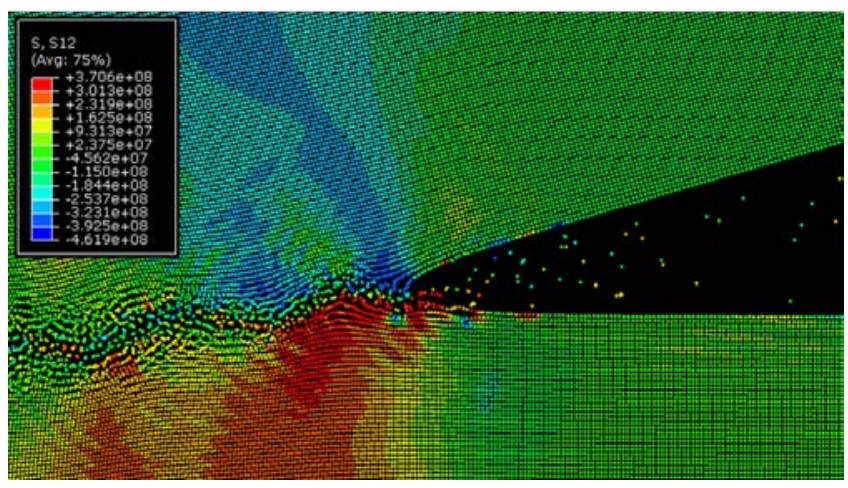

Figure 8:Predicted shear stress

Fig. 9 shows the temperature distribution at the interface. The results show a narrow region where the temperature is less than $400^{\circ} \mathrm{C}$. However, the temperature of the jetted material is above $400^{\circ} \mathrm{C}$ but still lower than the melting temperature of the Al6061-T6 $\left(\sim 653^{\circ} \mathrm{C}\right)$. Thus, the bonding was presumed to be a solid-state weld.

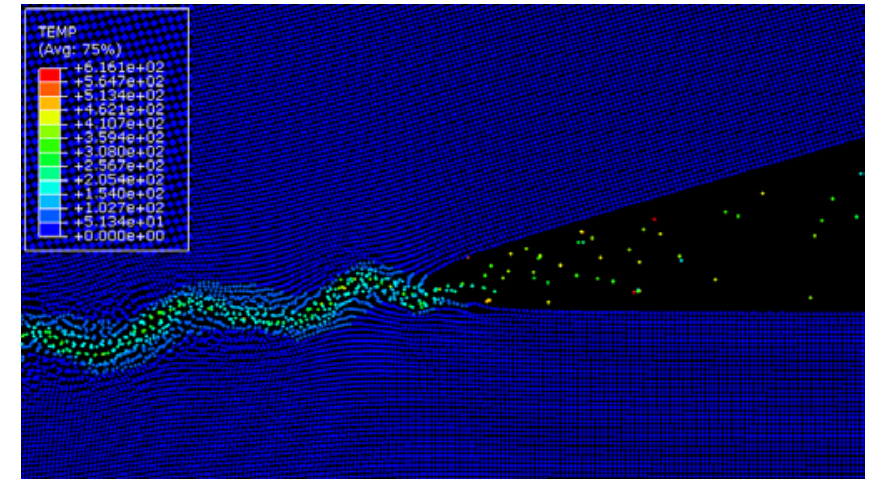

Figure 9:Predicted temperature at the interface

\subsection{Arbitrary Lagrangian-Eulerian (ALE)}

To contrast the SPH method for modeling the HVIW process, ALE numerical simulations were also conducted.To accurately model the impact process and emergent wavy morphology, a fine mesh was used near the contact point. On the other hand, to avoid instability due to the explicit dynamic nature of the code, the mesh size should be larger than $C_{d} \times \Delta t$, where $C_{d}$ is dilatational wave speed in the material and $\Delta t$ is the time increment. A mesh sensitivity study was performed to verify that the simulation results are not an artifact of the mesh density chosen. In the end, a structured quad mesh (i.e., $5 \mu \mathrm{m} \times 5 \mu \mathrm{m}$ ) was used in Region A (see Fig. 10). The thickness of this region is $25 \mu \mathrm{m}$ from the surface of the plates which was sufficient to capture impact region effects as was also confirmed with the sensitivity study. The remainder of the geometry in Regions B and C were automeshed with quaddominated elements. The element type was CPE4R (plane strain, four node, reduced integration), and 
a total of 153,279 elements were used in the model. Reduced integration elements assure that volumetric locking and excessive stiffness will not occur in the model. Also, to avoid spurious solutions due to the potentially flexible elements, hourglassing was controlled through the viscous approach. Since the high velocity impact process involves large deformations, adaptive meshing was used to minimize element distortion and allow the analysis to run to completion. Adaptive meshing could be either local or global. In this study to reduce the simulation cost, the adaptive domain was defined in Regions A and B of each plate (see Fig. 10).For adaptive meshing, the Initial Feature Angle Control (IFAC) was used as a boundary region smoothing algorithm [16].The other parameters were identical to Section 2.1.Seereference [7]for further information about the ALE method used.

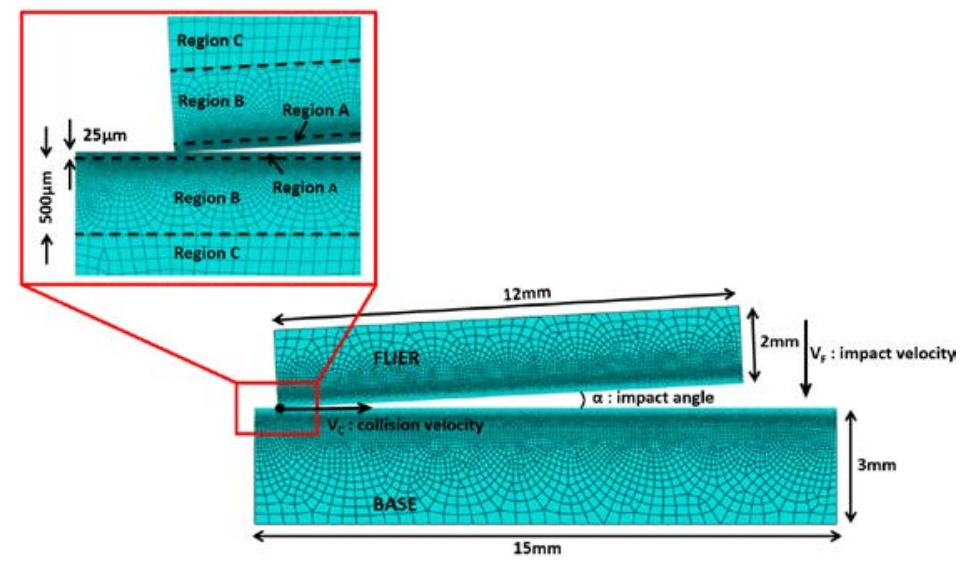

Figure 10:ALE model with varying mesh densities in three regions [7]

Fig. 11 shows the collision velocity fieldnear the contact zone. As is evident in the figure, a symmetric localized high velocity zone was produced again at the contact point in which the material close to the collision point of both the flier and base plates moves with the same velocity. The ALE confirms that the highest velocity vectors are at the collision point.The velocity of the collision point, $\sim 1150 \mathrm{~m} / \mathrm{s}$, is reasonably comparable to the velocity of the collision point from the SPH simulations, $\sim 1200 \mathrm{~m} / \mathrm{s}$ (see Fig. 7).

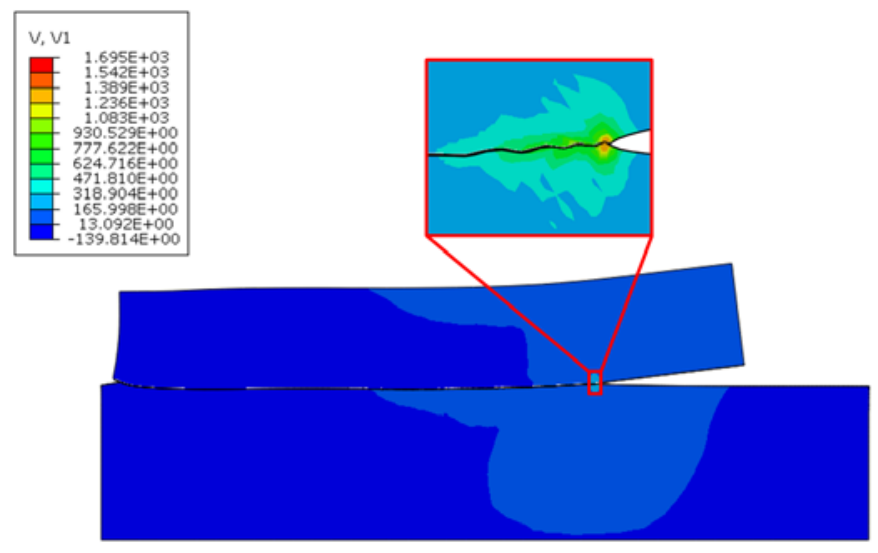

Figure 11:Predicted symmetric collision velocity distribution [7] 
Fig. 12 shows the shear stress where different signs were produced at the interface. The predicted shear stress at the interface by the ALE, 317MPa, is approximately $17 \%$ smaller than the SPH method, 370MPa.However, the value exceeded the shear yield stress of materialconfirming thatboth plates were plastically deformed.

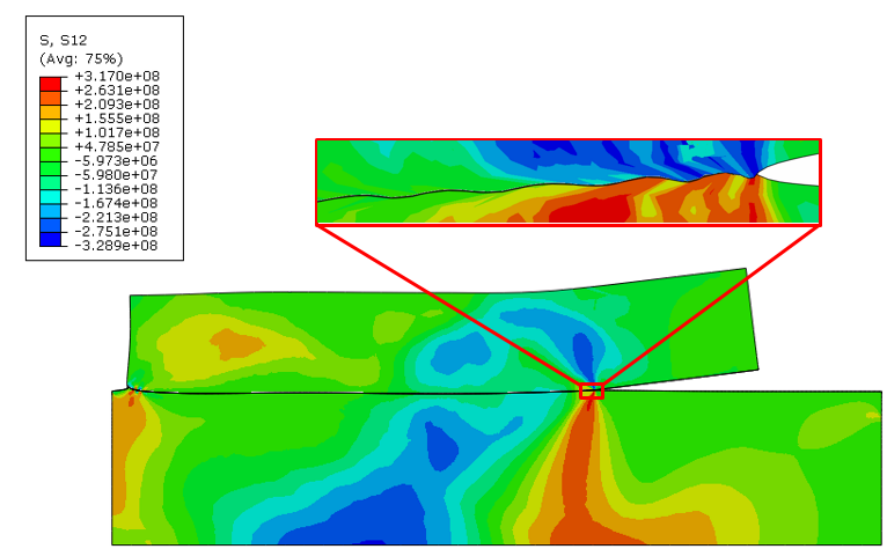

Figure 12:Predicted shear stress [7]

Fig. 13, shows the temperature distribution at the interface. The results show a narrow region $(\sim 20 \mu \mathrm{m})$ where the temperature value is $\sim 410^{\circ} \mathrm{C}$ (approximately 2 percent larger than the predicted value from the SPH method)which is lower than the melting temperature of the Al6061-T6 $\left(\sim 653^{\circ} \mathrm{C}\right)$.Thus, the bonding was presumed to be a solid state weld. This narrow region is where the maximum plastic deformation and collision velocity (see Fig. 11) occuras well. Note that in Fig. 13, the maximum temperature in the legend $\left(651^{\circ} \mathrm{C}\right)$ is for the temperature at the left corner of the model where the flier plate first contacts the base plate, not the weld interface.

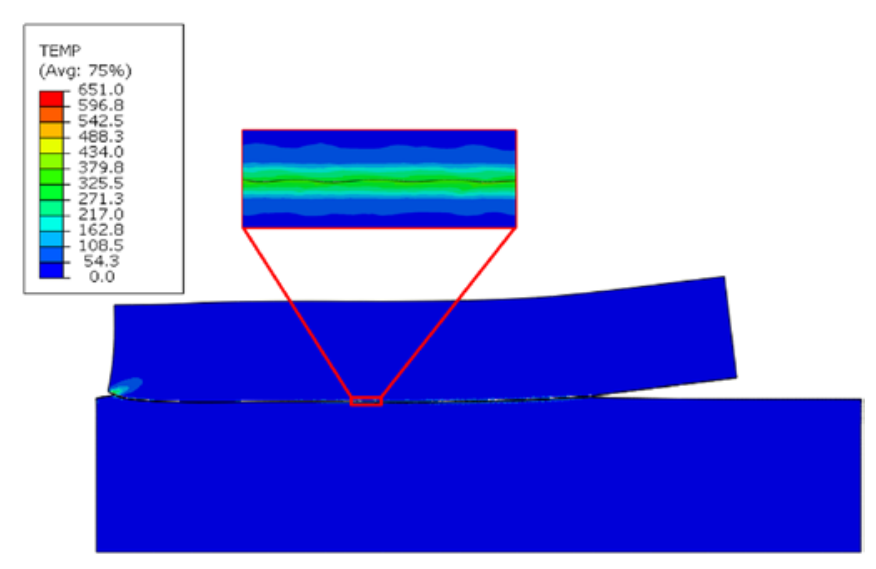

Figure 13:Predicted temperature at the interface [7] 


\section{Discussion}

To validate the accuracy of the SPH and ALE methods, an experimental test with a VFAW process wasconducted. As in the simulations, the material used for both plates was Al6061-T6, the thicknesses were $2 \mathrm{~mm}$ and $3 \mathrm{~mm}$ respectively for the flier and base plates. The experimental test was conducted by charging a Maxwell Magneform capacitor bank to $4.5 \mathrm{~kJ}$ which created the impact velocity of $350 \mathrm{~m} / \mathrm{s}$ as measured via Photon Doppler Velocimetry (PDV). The initial impact angle of $7^{\circ}$ was achieved by manually placing two $2.5 \mathrm{~mm}$ thick stand-offs with the horizontal distance (D) of $52.7 \mathrm{~mm}$ between them. See Fig. 14 for the experimental setup.

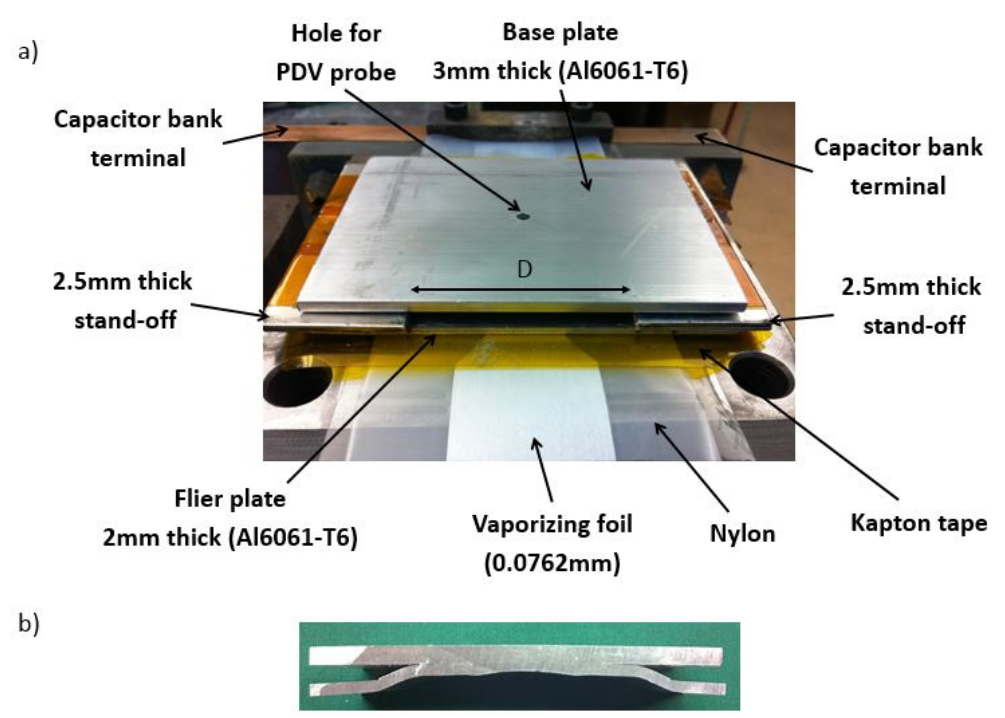

Figure 14:Experimental setup, a) location of the flier, base plate, and vaporizing foil, b) welded workpiece

Fig. 15shows the cross section of the weld interface with the wavy morphology. As predicted from the SPH simulations (Fig. 9) and ALE (Fig. 13) analyses, there is no indication of a molten zone for the case that welded with the impact velocity of $\mathrm{V}_{\mathrm{F}}=350 \mathrm{~m} / \mathrm{s}$, and the initial preset impact angle of $\alpha=7^{\circ}$. However, there were some small surface cracks and voids found at the interface. These were produces due to the significant plastic deformation. In terms of tracking the interface, both methods clearly captured the interfacial wavy morphology. The average of the wavelength measured from the experimental test was $90 \mu \mathrm{m}$, while, for the SPH and ALE methods, these values were $85 \mu \mathrm{m}$ and $80 \mu \mathrm{m}$, respectively.

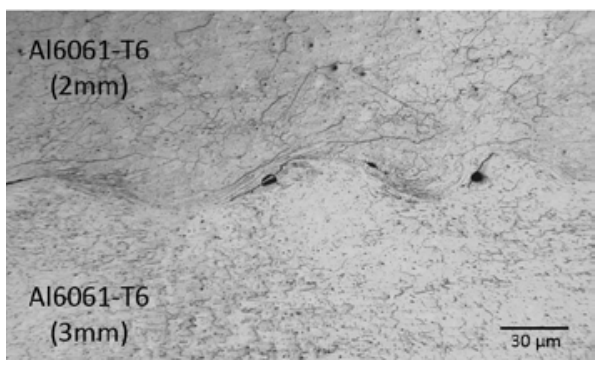

Figure 15:The interface between two Al6061-T6 plates 
As shown in Section 2, both methods were capable of modeling the process. As mentioned previously, jetting maybe a prerequisite for welding to occur (e.g., by removing any surface contaminant and the oxide material). However, this idea has not been explicitly evaluated with respect to the depth of the material in the plates being jetted. Although the SPH method was computationally more expensive than the ALE method, the jetting phenomena could only be simulated through the SPH method. As shown in Fig. 6, seemingly, the jet was propagated along the joining interface, and removed approximately $5 \mu \mathrm{m}$ of the SPH mesh away. Table 2 compares quantities and characteristics between the two numerical simulation methods.

\begin{tabular}{ccc}
\hline & SPH & ALE \\
\hline Ability to track the interface & Yes & Yes \\
\hline Ability to capture the jetting phenomena & Yes & No \\
\hline Computational time (secondx10 $)^{*}$ & 432 & 259.2 \\
\hline Storage $(\mathrm{GB})$ & 5.7 & 1.68 \\
\hline Collison velocity $(\mathrm{m} / \mathrm{s})$ & 1200 & 1150 \\
\hline Shear stress $(\mathrm{MPa})$ & 370 & 317 \\
\hline Temperature $\left({ }^{\circ} \mathrm{C}\right)$ & 400 & 410 \\
\hline Wavelength $(\mu \mathrm{m})$ & 85 & 80 \\
\hline Amplitude $(\mu \mathrm{m})$ & 12 & 10 \\
\hline
\end{tabular}

Table 2: Comparison between SPH and ALE methods

* 2 Quad Core (8 virtual CPUs), 32GB RAM SWAP server (parallelization set to 7 CPUs)

\section{Conclusion}

In this paper, two numerical simulation methodsfor modeling HVIW processes, the SPH and ALE techniques, were investigated and compared to experimental results.The wavy morphology, predicted temperature values across the interface, velocity of the material at the collision point and the shear stress at the interface were in good agreement for these two FEA methods and the experimental data.But only using the SPH method was jetting phenomenon and the composition of the jetted material able to be simulated. The accurate modeling of this jetting phenomenon will allow further investigation of the design space compared to trial-and-error experimental investigations in particular for different material systems.Generally,SPH method is less accurate than the pure Lagrangian FEA method especially when the deformation is not severe. Moreover, when the material is in a state of tensile stress, the particle motion may become unstable.To validate the accuracy of the SPH method, in the future, the composition of the jetted material from the model will be compared with the jetted material that will be collected in experiments by placing a witness block perpendicular to the direction of weld. This will provide data related to the material which is jetted from the process, e.g., the oxide layer on the Aluminum, and thus not a part of the final interface weld. The collected jetted material will be characterized in terms of mass and composition.

\section{Acknowledgements}

Supportfrom the U.S. National Science Foundation under Grants CMMI-0928319 and CMMI1537471 is gratefully acknowledged. 


\section{References}

[1] Li, Y., Lin, Z., Jiang, A., Chen, G. Use of high strength steel sheet for lightweight and crashworthy car body. Materials \& design2003; 24(3): 177-182.

[2] Shaw, J. R. and Zuidema, B. K.New high strength steels help automakers reach future goals for safety, affordability, fuel efficiency and environmental responsibility. SAE Technical Paper, 2001, No. 2001-01-3041.

[3] Kacar, R. and Acarer, M. An investigation on the explosive cladding of 316L stainless steeldin-P355GH steel. Journal of Materials Processing Technology2004; 152(1): 91-96.

[4] Zhang, P. Joining enabled by high velocity deformation, The Ohio State University, 2003.

[5] Kochan, A. Magnetic pulse welding shows potential for automotive applications. Assembly Automation2000; 20(2): 129-132.

[6] Shribman, V., Gafri, O., Livshitz, Y. Magnetic Pulse Welding \& Joining-A New Tool for the Automotive Industry. SAE Technical Paper, 2001, No. 2001-01-3408.

[7] Nassiri, A., Chini, G., Vivek, A., Daehn, G., Kinsey, B. Arbitrary Lagrangian-Eulerian finite element simulation and experimental investigation of wavy interfacial morphology during high velocity impact welding. Materials \& Design2015; 88: 345-358.

[8] Nassiri, A., Chini, G., Kinsey, B. Spatial stability analysis of emergent wavy interfacial patterns in magnetic pulsed welding. CIRP Annals-ManufacturingTechnology2014; 63(1): 245-248.

[9] Nassiri A, Kinsey B, Chini G. Shear instability of plastically-deforming metals in highvelocity impact welding. Journal of the Mechanics and Physics of Solids. 2016, doi:10.1016/j.jmps.2016.06.002.

[10] Manikandan, P., Hokamoto, K., Deribas, A. A., Raghukandan, K., Tomoshige, R. Explosive welding of titanium/stainless steel by controlling energetic conditions. Materials transactions2006; 47(8): 2049-2055.

[11] Nassiri, A. Investigation of Wavy Interfacial Morphology in Magnetic PulsedWelding: Mathematical Modeling, Numerical Simulations and ExperimentalTests. University of New Hampshire, 2015.

[12] Vivek, A., Hansen, S. R., Liu, B. C., Daehn, G. S. Vaporizing foil actuator: A tool for collision welding. Journal of Materials Processing Technology2013; 213(12): 2304-2311.

[13] Mousavi, A. A. and Al-Hassani, S. T. S. Numerical and experimental studies of the mechanism of the wavy interface formations in explosive/impact welding. Journal of the Mechanics and Physics of Solids2005; 53(11): 2501-2528.

[14] Sarrate, J., Huerta, A., Donea, J. Arbitrary Lagrangian-Eulerian formulation for fluid-rigid body interaction. Computer Methods in Applied Mechanics and Engineering2001; 190(24): 3171-3188.

[15] Nazem, M., Sheng, D., Carter, J. P., Sloan, S. W. Arbitrary Lagrangian-Eulerian method for large-strain consolidation problems.International journal for numerical and analytical methods in geomechanics2008; 32(9): 1023-1050.

[16] Wen, Q., Guo, Y. B., Todd, B. A. An adaptive FEA method to predict surface quality in hard machining. Journal of materials processing technology2006; 173(1): 21-28.

[17] Hayhurst, C. J. and Clegg, R. A. Cylindrically symmetric SPH simulations of hypervelocity impacts on thin plates. International Journal of Impact Engineering1997; 20(1): 337-348.

[18] Lenaerts, T., Adams, B., Dutré, P. Porous flow in particle-based fluid simulations. ACM Transactions on Graphics (TOG) 2008; 27(3):49

[19] Xu, X., Ouyang, J., Yang, B., Liu, Z. SPH simulations of three-dimensional non-Newtonian free surface flows. Computer Methods in Applied Mechanics and Engineering2013; 256: 101-116. 
[20] Muller, M., Schirm, S., Teschner, M. Interactive blood simulation for virtual surgery based on smoothed particle hydrodynamics. Technology and Health Care-European Society for Engineering and Medicine2004; 12(1): 25-32.

[21] Monaghan, J. J. Smoothed particle hydrodynamics and its diverse applications. Annual Review of Fluid Mechanics2012; 44: 323-346.

[22] Bonora N. and Brown, E. Numerical Modeling of Materials Under Extreme Conditions. Springer US, 2014.

[23] Ravichandran, G., Rosakis, A.J., Hodowany, J., Rosakis, P. On the conversion of plasticwork into heat during high-strain-rate deformation, Proceedings of the Shock Compression of Condensed Matter, American Institute of Physics, Atlanta, GA,2002,pp. 557-562.

[24] Abaqus Analysis User's Manual Version 6.13, Volume II, Providence, RI, 2014.

[25] Brasher, D. G. and Butler, D. J.Explosive welding: principles and potentials. Advanced Materials and Processes1995; 147(3).

[26] Alexander, M. R., Thompson, G. E., Zhou, X., Beamson, G., Fairley, N. Quantification of oxide film thickness at the surface of aluminium using XPS. Surface and interface analysis2002; 34:485-489. 